\title{
Observations of Binary Protostellar Systems
}

\author{
Ralf Launhardt \\ Max Planck Institute for Astronomy, Königstuhl 17, 69117 Heidelberg, \\ Germany, email: rl@mpia.de
}

\begin{abstract}
.
A major gap in our understanding of star formation concerns the origins of binary stars although there is growing evidence that most stars form in binary and multiple systems. While some theoretical predictions of fragmentation models are indirectly supported by statistical studies of evolved binary stars at shorter wavelengths, direct observations of the formation phase became only possible with the advance of large millimeter interferometers. Molecular line spectroscopy and dust continuum observations at high angular resolution can address some of the key questions in binary star formation theories. Observing methods and results of recent studies of binary protostellar systems are reviewed in this paper.
\end{abstract}

\section{Introduction}

Multiple stellar systems have been observed in all stages of evolution and there is growing evidence of frequent multiplicity already in the protostellar stage. Both the high frequency of binary systems and the recognition that binarity originates in the formation phase let us realize that fragmentation plays an important role in the star formation process and that binary formation may be the "standard mode" of star formation. However, our current knowledge on the formation of binary stars relies on observations of main sequence (MS) and pre-main sequence (PMS) stars and the constraints they put on the models (e.g., Clarke 2001; Ghez 2001). Observations of protostellar stages where long hampered by the low angular resolution of $\mathrm{mm}$ telescopes and the results where mostly interpreted in terms of single star formation. Only the recent advance of large mm interferometers has enabled us to directly observe the formation phase of binary stars. The most recent compilation of observational results and theoretical models on the formation of binary stars can be found in the proceedings of IAU Symposium 200 (Zinnecker \& Mathieu 2001). Observations of protobinary systems in particular where summarized by Mundy et al. (2001). Theoretical aspects on the origin of binary stars were recently reviewed by Tohline (2002).

\section{Facts and open questions about binary stars and their formation}

Statistical properties of binary stars are still subject to extensive ongoing studies (e.g., Köhler \& Brandner 2001; Guenther et al. 2001; Kaltenegger et al. in prep.). Some of the most important results of these and earlier studies are: 
(1) Coeval formation: The components in PMS binary systems have similar ages implying coeval formation (White \& Ghez 2001).

(2) Frequency: The overall binary frequency among MS stars is of order $50 \%$ (Duquennoy \& Mayor 1991). The binary frequency among PMS stars is much higher (see Duchêne 1999 and references therein). The numbers are consistent with the possibility that all stars form in multiple systems and single stars are the result of disintegration (Sterzik \& Durisen 1998; Larson 2001). However, differences between star-forming regions imply a possible dependence on the initial conditions in the molecular cloud due to temperature or turbulence.

(3) Periods: The distribution of orbital periods is very broad and nearly flat with a median of $\approx 180$ yr $(a \approx 35$ AU; Duquennoy \& Mayor 1991 ; see Fig. 2a). (4) Mass Ratios: The distribution of mass ratios for PMS and MS binaries with $a<0.2 \mathrm{AU}$ appears to be flat, i.e., unequal masses are more frequently observed than equal masses (Woitas et al. 2001; Halbwachs et al. 2003).

Different formation scenarios have been proposed, of which the classical ideas of capture and fission are no longer considered major processes. While there is widespread agreement that fragmentation during the formation phase is the most important mechanism that leads to binary stars, the exact when, where, why, and how are still under debate. Prompt fragmentation of rotationally flattened cloud cores with initially flat density profiles, immediately after a phase of free-fall collapse, appears to be the most promising scenario. The outcome of this process is thought to be controlled mainly by the distribution of angular momentum of the infalling material (e.g., Bate \& Bonnell 1997). Delayed fragmentation of massive accretion disks may lead to very close systems (Bonnell et al. 1991). Very wide binaries may also form from separately collapsing cores that where formed by prompt initial fragmentation of a larger cloud (e.g. Pringle 1989). But many of these systems may not survive as binaries to the main sequence.

Constraints on binary formation models so far come from observations of MS and PMS binaries and from numerical simulations. However, the link between initial conditions in a molecular cloud and the final star systems is missing. Furthermore, as multiple systems certainly undergo dynamical evolution, important information about the formation phase is lost in the final systems. Direct observations of protostars should therefore address the following questions:

(i) How common is binarity/multiplicity in the protostellar phase?

(ii) How many and which protostellar cores fragment in the collapse phase? Where do we observe prompt initial fragmentation and separately collapsing cores? What are the initial distributions of separations and mass ratios?

(iii) How is angular momentum distributed? Are there differences between cores forming binaries and those forming single stars?

(iv) How does the collapse proceed after fragmentation? Do accretion rates depend on separation, mass ratios, angular momentum?

Points ( $i$ ) and (ii) can already be addressed, at least for the wide part of the separation distribution. However, the numbers are still small. Answering point (iii) is more difficult, but a start is done (Sect. 4.2). Point (iv) requires extensive modeling, and both very high angular resolution and good $u v$ coverage. However, suitable tracers for the central collapse region may turn out to be a problem. Reliable observational results do not exist yet (Sects. 3 and 4.2). 


\section{Tracers and observing methods}

Protostars are deeply embedded in molecular cloud cores and are surrounded by opaque, infalling envelopes and accretion disks. All radiation emanating from the protostar is re-processed by the surrounding dust. Therefore, only emission from circumprotostellar material can usually be directly observed. The range of dust temperatures and gas densities involved in low-mass protostellar cores make observations of the thermal dust continuum emission from far-infrared to $\mathrm{mm}$ wavelengths as well as $\mathrm{mm}$ molecular line transitions most favorable. The list of tracers and observing methods for studying binary protostars includes:

(1) NIR: At near-infrared (NIR) wavelengths, scattered light from outflow cavities of protostars can be observed (e.g., CB 230, Fig 3a). More evolved systems can be directly observed in the NIR (Greene, this volume). Protostars also drive Herbig-Haro jets, which can be observed in shock-excited NIR lines. From the morphology of these flows, conclusions on the location, alignment, and orbital evolution of young binary systems can be drawn (Reipurth, this volume).

(2) MIR-FIR: Mid to far-infrared continuum emission traces hot dust in the immediate environment of the protostar. The strengths of the emission depends on the evolutionary stage and temperature. The low angular resolution of current instruments usually prohibits resolving binary protostars at these wavelengths. However, ISOCAM images could reveal the location of secondary components in two wide binary protostellar systems (CB 230, Fig. 3a; BHR 71, Bourke 2001).

(3) $M M$ continuum: The submm and $\mathrm{mm}$ dust continuum emission traces the bulk the dust mass in protostellar cores and accretion disks (Sect. 4.1.).

(4) Molecular lines: Molecular lines at $\mathrm{mm}$ wavelengths can be used to trace dense gas in protostellar cores. However, most lines become either optically thick or the molecules deplete rapidly onto grain surfaces in the centers of dense cores. Some optically thick lines with high critical densities can be used to observe infall motions (e.g., $\mathrm{CS}, \mathrm{HCO}^{+}$). Others can be used to derive rotation curves and other kinematic properties (e.g., $\mathrm{N}_{2} \mathrm{H}^{+}$) (Sect. 4.2.). Molecules like ${ }^{12} \mathrm{CO}$ and ${ }^{13} \mathrm{CO}$ can be used to trace molecular outflows. When observed at high angular resolution, the location of the driving sources and their relative orientation in the plane of the sky can be derived (Sect. 4.3.).

The largest current-day single-dish $\mathrm{mm}$ telescopes provide angular resolutions of $\approx 10^{\prime \prime}$ at $\lambda 1 \mathrm{~mm}$, which at a distance of $200 \mathrm{pc}$ corresponds to $2000 \mathrm{AU}$. This is far beyond the the peak of the binary separation distribution (see Fig. 2a). Most information about binary protostellar systems can therefore only be revealed with $\mathrm{mm}$ interferometers, which currently provide angular resolutions of up to $0.5^{\prime \prime}$ and thus come close to the maximum of the separation distribution.

\section{Observations of binary protostars}

\subsection{Results from interferometric millimeter continuum observations}

During the past few years, interferometric mm dust continuum observations lead to the discovery of several binary protostellar systems. Table 1 lists the known protobinary systems and Fig. 1 shows two examples of synthesized mm continuum images. Here both Class 0 protostars and deeply embedded Class I sources are included. For reasons given below, only systems with a projected separa- 

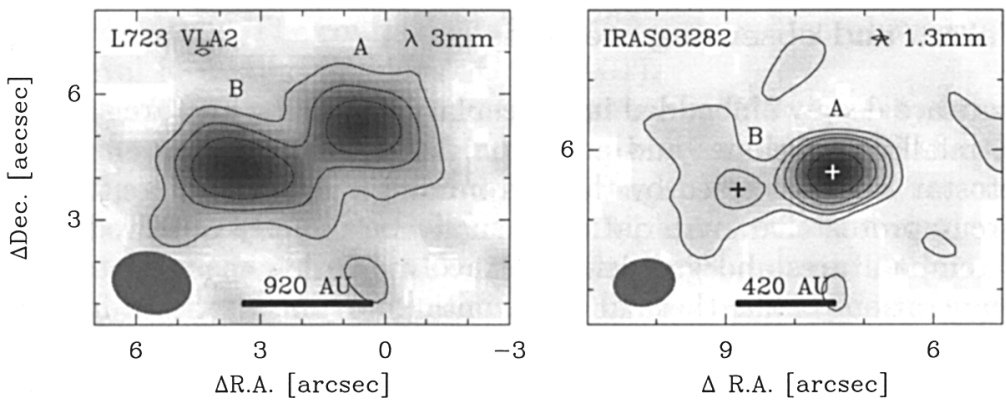

Figure 1. Interferometric mm continuum maps of the binary protostars L723 VLA2 and IRAS 03282, obtained with the Owens Valley millimeter array (Launhardt et al. in prep.). Synthesized beam sizes are shown as grey ovals. Contours start at $3 \sigma(\sigma=0.3 \mathrm{mJy} /$ beam at $3 \mathrm{~mm}$ for $\mathrm{L} 723$ and $2.5 \mathrm{mJy} /$ beam at $1.3 \mathrm{~mm}$ for IRAS 03282).

Table 1. Known protobinary systems and circumstellar mass ratios

\begin{tabular}{lcccl}
\hline Source & Class & Sep. $[\mathrm{AU}]$ & $M_{2} / M_{1}$ & Reference \\
\hline L1551 IRS5 & I & 50 & 0.4 & Looney et al. 1997 \\
VLA1623 & 0 & 180 & 1.0 & Looney et al. 2000 \\
L1551-NE & 0 & 230 & 0.4 & Moriarty-Schieven et al. 2000 \\
IRAS03282+3035 & 0 & 420 & 0.23 & Launhardt et al. in prep. \\
CB188 & 0 & 510 & 0.5 & Launhardt et al. in prep. \\
NGC1333 IRAS4 A & 0 & 600 & 0.25 & Looney et al. 2000 \\
IRAS16293-2422 & 0 & 820 & 0.8 & Mundy et al. 1992 \\
L723 VLA2 & 0 & 920 & 0.8 & Launhardt et al. in prep. \\
SVS13 A & I? & 1840 & $0.2^{*}$ & Looney et al. 2000 \\
L1448 IRS3 & 0 & 2400 & 0.18 & Looney et al. 2000 \\
L1527 & 0 & 2800 & 0.4 & Fuller et al. 1996 \\
BHR71 IRS & 0 & 3400 & $0.1^{*}$ & Bourke 2001 \\
NGC1333 IRAS4 B/C & 0 & 3720 & 0.27 & Looney et al. 2000 \\
CB230 & I(0?) & 3800 & 0.1 & Launhardt 2001 \\
SVS13 A/B & $0 / \mathrm{I}$ & 3840 & 0.77 & Looney et al. 2000 \\
CG30 & 0 & 4000 & 0.7 & Launhardt et al. 2000 \\
\hline \hline
\end{tabular}

${ }^{*}$ These values are very uncertain guesses

tion of $\leq 4000 \mathrm{AU}$ are listed. Figure $2 \mathrm{a}$ demonstrates that protobinary systems have been detected at all separations accessible to current $\mathrm{mm}$ interferometers. However, due to the small numbers and strong detection bias towards wider separated systems, a separation distribution cannot be derived yet.

Both the surveys by Looney et al. (2000) and Launhardt et al. (in prep.) suggest that systems with a separation of less than $\approx 4000 \mathrm{AU}$ have a common envelope while wider systems have separate envelopes. However, the extent to which the envelopes are traced depends on the sensitivity, angular resolution, and $u v$ weighting. The only solid conclusion that can be drawn at this time is that the observations do not contradict the assumption that protostars with separations smaller than the typical size of a prestellar core accrete material from a common envelope. In order to put more solid constraints on formation models, we have to observe spatially resolved infall motions and reconstruct the 

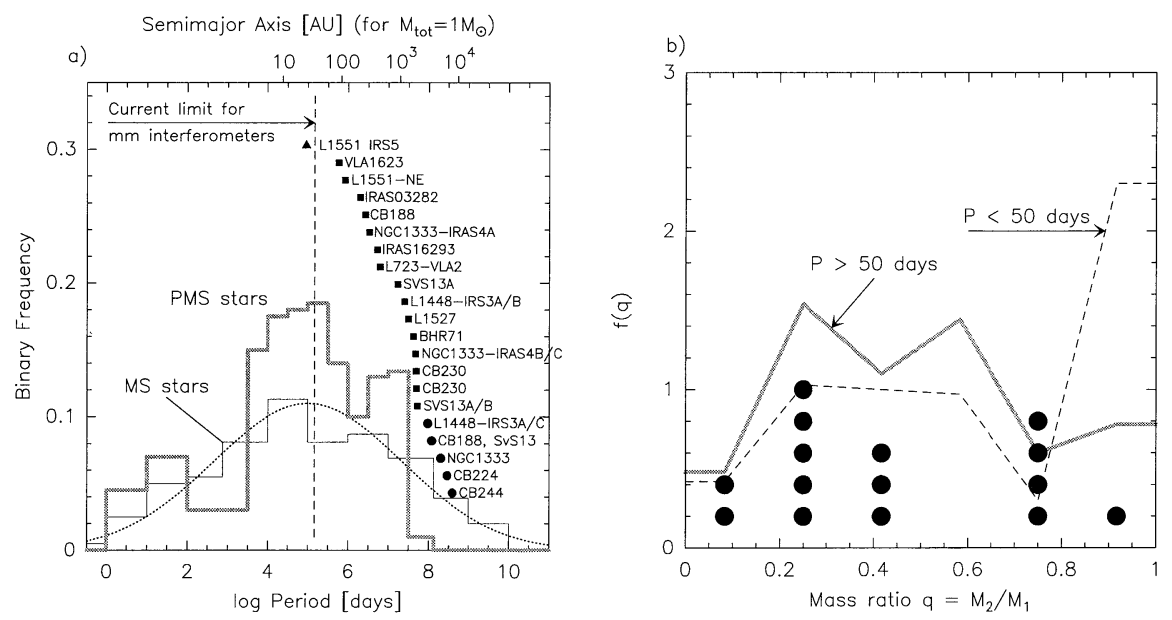

Figure 2. (a) Period/separation distribution for low-mass binaries (Duquennoy \& Mayor 1991; Mathieu 1994; Simon et al. 1995). Known protobinary systems are shown according to their separation whithout implying a frequency distribution. Circles mark systems with separate envelopes. (b) Distribution of mass ratios in MS binaries (Halbwachs et al. 2003). Black dots show circumstellar mass ratios of known protobinary systems (Table 1).

3-D density structure. Both tasks are extremely difficult and the latter one cannot be realized with the standard 1-D $u v$ analysis of interferometric data.

Absolute masses derived from mm continuum images are very uncertain and often cannot be compared because different observing and analysis methods trace different amounts of material. Relative masses are more reliable although they are often just scaled with the flux density without detailed modeling of the temperature structure which might be different for different protostar masses, accretion rates, and disk sizes. Table 1 lists the circumstellar mass ratios of all known protobinary systems under the assumption that the mass is proportional to the mm flux density. Note that the circumstellar mass ratio does not necessarily reflect the mass ratio of the hydrostatic cores nor that of the final stars. Nevertheless, a first attempt to compile mass ratios of binary protostars shows a rather flat distribution like that of more evolved long-period binary stars (Fig. $2 \mathrm{~b}$ ). Since equal-mass systems are easier to detect, this is not an observational effect. Quantitatively, we can draw the preliminary conclusion that at least two thirds of protobinary systems have circumstellar mass ratios below 0.5 while one third or less have mass ratios higher than 0.7 .

\subsection{Kinematics of protostellar cores}

Obtaining detailed kinematic information on, e.g., infall motions and the distribution of angular momentum in protostellar cores is one of the key issues in constraining models of binary star formation. However, the revelation of such information is hampered by nature. Most of the easily observable tracer molecules either become optically thick or deplete in the central regions of such cores. Outflows impose further complications by the destruction of certain molecules (e.g., 

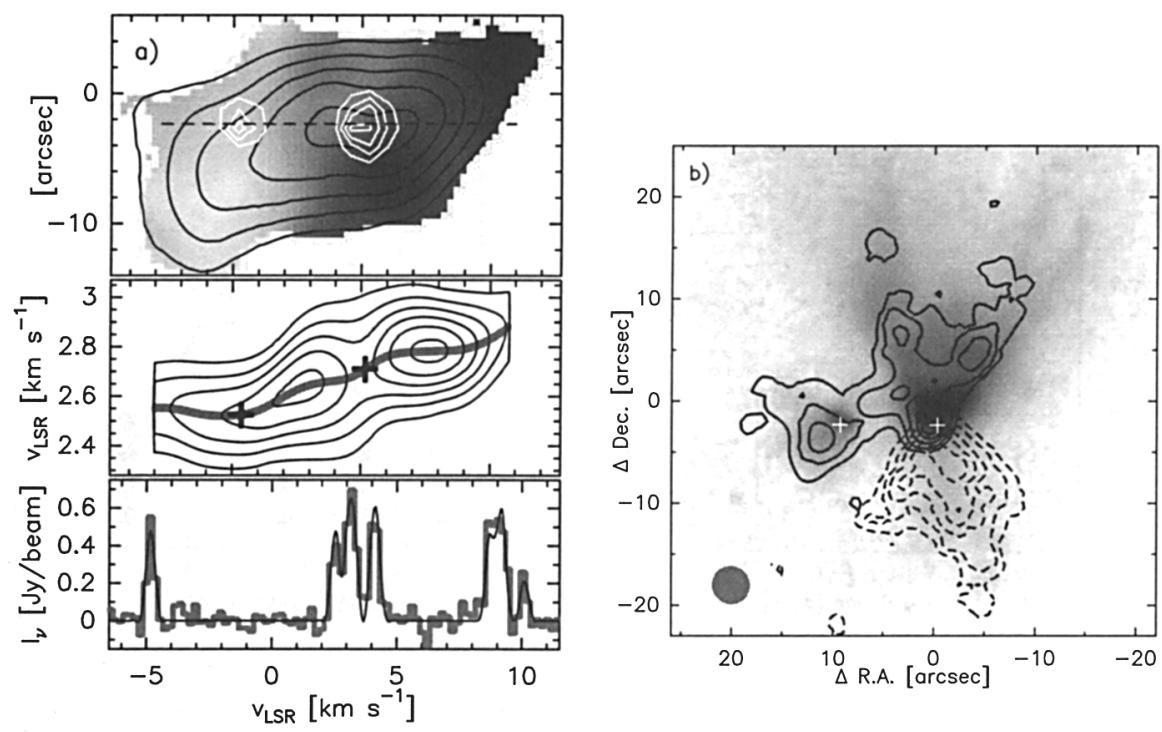

Figure 3. (a) Kinematic structure of the CB 230 core. Top: integrated $\mathrm{N}_{2} \mathrm{H}^{+}$map (black contours) and mean velocity map (grey-scale, white to black: 2.4 to $2.9 \mathrm{~km} \mathrm{~s}^{-1}$ ). White contours show the MIR sources observed with ISOCAM. Center: position-velocity diagram along the dashed line in top panel. Bottom: $\mathrm{N}_{2} \mathrm{H}^{+}(1-0)$ spectrum and fit. (b) ${ }^{13} \mathrm{CO}(1-0)$ map of the double outflow in CB 230, overlayed on a grey-scale image of the NIR reflection nebula. Solid and dashed contours show blue-shifted $\left(0.7-2.5 \mathrm{~km} \mathrm{~s}^{-1}\right)$ and redshifted line wing emission (3.1-5.2 $\mathrm{km} \mathrm{s}^{-1}$ ) (Launhardt 2001; in prep.).

$\mathrm{N}_{2} \mathrm{H}^{+}$), the local gas-phase enhancement of others (e.g., SiO), the creation of cavities, and interactions with the envelope. Radiation from such molecules often provides misleading kinematic information.

While for these reasons spatially resolved information about infall motions in binary protostars is not yet available, first rotation curves have been be derived from high-resolution observations of $\mathrm{N}_{2} \mathrm{H}^{+}$(Launhardt 2001). $\mathrm{N}_{2} \mathrm{H}^{+}$is easily detroyed by $\mathrm{CO}$ and $\mathrm{H}_{2} \mathrm{O}$, but it is abundent where these molecules are frozen out, i.e., in the cold, dense, and quiescent regions of prestellar and protostellar cores (Bergin \& Langer 1997; Bergin et al. 2001; Doty et al. 2002). For the same reason it is depleted in the very central region near the protostar, in the outer envelopes, and in outflows. $\mathrm{N}_{2} \mathrm{H}^{+}$is therefore a good tracer of quiescent dense gas in protostellar cores. By simultaneously fitting all seven lines of its $J=1-0$ hyperfine structure complex, very accurate velocity fields can be derived.

Figure $3 \mathrm{a}$ shows the velocity field of the protostellar double core in the Bok globule CB 230 derived from interferometric $\mathrm{N}_{2} \mathrm{H}^{+}$observations. To first order, it can be interpreted as solid-body rotation of the entire cloud core about an axis perpendicular to the connecting line between the two embedded protostars. The specific angular momentum at the location of the protostars is then $j \sim$ $3 \times 10^{16} \mathrm{~m}^{2} \mathrm{~s}^{-1}$ (Launhardt 2001). Similar observations, although with somewhat higher uncertainty, exist for L723 VLA2 and IRAS 03282 (Launhardt et al. in prep.). Figure 4 shows the evolution of specific angular momentum and size 


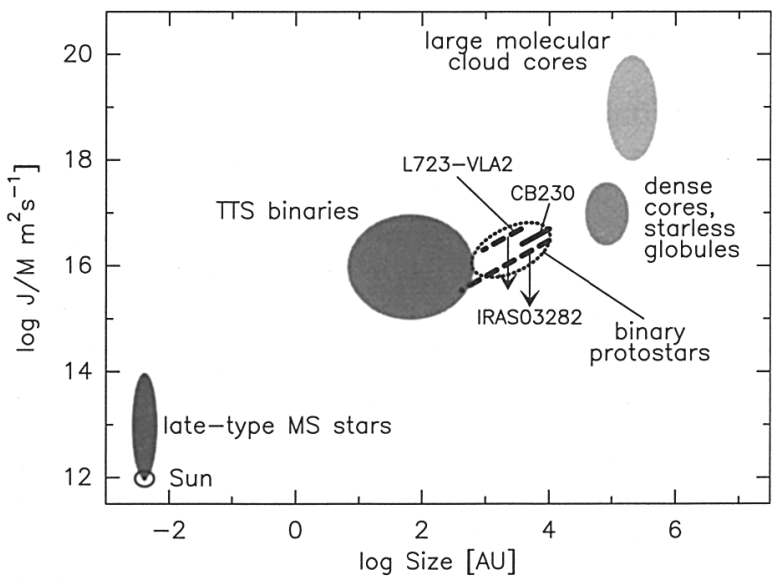

Figure 4. Evolution of specific angular momentum (adapted from Kane \& Clemens 1997; binary protostars: Launhardt et al. in prep.). The lines for the three binary protostars mark the range of specific angular momentum at different size scales in these cores.

from molecular cloud cores to stars. In this diagram, binary protostars close the gap between prestellar cores and T Tauri binary systems. It also becomes clear that most of the initial angular momentum of a protostellar core is transformed into orbital angular momentum of the resulting binary star system.

\subsection{High angular resolution observations of molecular outflows}

High-resolution observations of accelerated gas near the outflow centers can also reveal multiplicity of the driving sources, their location, as well as relative alignment. While extended $\mathrm{CO}$ emission at the rest velocity of the cloud is often resolved out in interferometer maps, well-structured outflow features are visible in the line wing emission. If binarity among protostars occurs as frequent as among PMS stars, one would excpect to find many multiple protostellar outflows. However, multipolar outflows have been observed in only a handful of sources. Possible reasons are: (i) Outflow-envelope interactions together with resolving out extended emission make the interpretation of interferometric outflow maps very complicated. (ii) If the protostellar jets (and disks) are aligned, they may drive one common large-scale outflow. (iii) The outflow momentum flux of protostars correlates well with the circumstellar envelope mass (Bontemps et al. 1996). Since binary protostars appear to have preferentially very unequal envelope masses (see Fig. 2b), their relative outflow strengths are expected to be also quite different. Thus, the weaker outflow may often not have been detected. The detections of an equal-mass protobinary system at the origin of the large quadupolar outflow in L 723 (Lee et al. 2002) and of a weak secondary outflow from the lower-mass component in the unequal-mass protobinary CB 230 (Fig. 3a; Launhardt 2001) confirm this trend. However, high-resolution outflow data so far exist only for very few systems and it is too early to derive correlations between alignment, relative outflow strengths, separation, and mass ratios. 


\section{Summary and outlook}

Direct observations of binary protostars have only become possible with the recent advance of large mm interferometers. Although current instruments do not provide the angular resolution required to routinely study size scales comparable to typical binary separations, a number of wider protobinary and multiple systems, some of them hierarchical, have been identified in the past few years. Due to the large effort and observing time required, the numbers are still small, detection biases are strong, and statistically significant conclusions cannot be drawn yet. However, a start is made and first trends are established:

1. Binarity/multiplicity is frequent among protostellar systems. All separations accessible to current instruments have been observed. However, due to the small numbers and the limited angular resolution, no reliable separation distribution or total binary frequency can be derived yet.

2. The circumstellar mass ratio distribution of (wide) binary protostars appears to be flat like that of more evolved long-period binary stars. Quantitatively, we can draw the preliminary conclusion that at least two thirds of protobinary systems have circumstellar mass ratios below 0.5 while one third or less have mass ratios higher than 0.7.

3. Despite frequent multiplicity, detections of multipolar protostellar outflows are rare. It is not clear yet whether this is due to preferential alignment of disks and angular momenta or due to very unequal outflow strengths.

4. In the evolution of specific angular momentum, binary protostars close the gap between prestellar cores and T Tauri binary systems. No significant differences between different systems can be seen yet. However, such information is only available for very few systems. The next steps on the road should be both to derive rotation curves and angular momenta for more binary protostars and to study the internal distribution of angular momentum in selected single and binary protostellar cores.

Both molecular line studies and chemical evolution models let us realize that there might be serious lack of reliable tracers of gas kinematics in the centers of protostellar cores where binary stars form. Better instruments alone may not help here, we also have to think about new methods and tracers. In the near future, the $3 \mathrm{~mm}$ upgrade of the Australia Telescope Compact Array (ATCA) will help to increase the number known protobinary systems in the southern sky. The Submillimeter Array (SMA) will help to identify more reliable tracers for the inner collapse region. The Combined Array for Research in Millimeter-wave Astronomy (CARMA) will, for the first time, allow efficient snapshot observations at subarcsec resolution and thus help to derive separations, mass ratios and rotation curves for a larger number of protobinary systems. Although these instruments will provide angular resolutions of down to $0.1^{\prime \prime}$ under best conditions, routine observations at better than 50 mas are required to study in detail statistically significant numbers of protostars at size scales which are more typical for binary star formation. ALMA, with an angular resolution of down to 10 mas, is thus the intrument which we expect to bring the breakthrough in our knowledge on the details of the star formation process. It may, however, also raise new, unexpected questions. 


\section{References}

Bate, M. R., \& Bonnell, I. A. 1997, MNRAS, 285, 33

Bergin, E. A., \& Langer, W. D. 1997, ApJ, 486, 316

Bergin, E. A., Ciardi, D. R., Lada, C. J., Alves, J., \& Lada, E. A. 2001, ApJ, 557,209

Bonnell, I., Martel, H., Bastien, P., Arcoragi, J., \& Benz, W. 1991, ApJ, 377, 553

Bontemps, S., André, P., Terebey, S., \& Cabrit, S. 1996, A\&A, 311, 858

Bourke, T. L. 2001, ApJ, 554, L 91

Clarke, C. J. 2001, see Zinnecker \& Mathieu 2001, 346

Doty, S. D., van Dishoeck, E. F., van der Tak, F. F. S., \& Boonman, A. M. S. 2002, A\&A, 389, 446

Duchêne, G. 1999, A\&A, 341, 547

Duquennoy, A., \& Mayor, M. 1991, A\&A, 248, 485

Fuller, G. A., Ladd, E. F., \& Hodapp, K.-W. 1996, ApJ, 463, L97

Ghez, A. M. 2001, see Zinnecker \& Mathieu 2001, 210

Guenther, E., Joergens, V., Neuhäuser, R., et al. 2001, see Zinnecker \& Mathieu 2001, 165

Halbwachs, J. L., Mayor, M., Udry, S., \& Arenou, F. 2003, A\&A, 397, 159

Kane, B. D. \& Clemens, D. P. 1997, AJ, 113, 1799

Köhler, R., \& Brandner, W. 2001, see Zinnecker \& Mathieu 2001, 147

Launhardt, R. 2001, see Zinnecker \& Mathieu 2001, 117

Larson, R. B. 2001, see Zinnecker \& Mathieu 2001, 93

Lee, C., Mundy, L. G., Stone, J. M., \& Ostriker, E. C. 2002, ApJ, 576, 294

Looney, L. W., Mundy, L. G., \& Welch, W. J. 1997, ApJ, 484, L157

Looney, L. W., Mundy, L. G., \& Welch, W. J. 2000, ApJ, 529, 477

Mathieu, R. D. 1994, ARA\&A, 32, 465

Moriarty-Schieven, G. H., Powers, J. A., Butner, H. M., Wannier, P. G., \& Keene, J. 2000, ApJ, 533, L143

Mundy, L. G., Wootten, A., Wilking, B. A., Blake, G. A., \& Sargent, A. I. 1992, ApJ, 385, 306

Mundy, L. G., Looney, L. W., \& Welch W. J. 2001, see Zinnecker \& Mathieu 2001,136

Pringle, J. E. 1989, MNRAS, 239, 361

Simon, M., Ghez, A. M., Leinert, C., et al. 1995, ApJ, 443, 625

Sterzik, M. F., \& Durisen, R. H. 1998, A\&A, 339, 95

Tohline, J. E. 2002, ARA\&A, 40, 349

White, R. J., \& Ghez, A. M. 2001, ApJ, 556, 265

Woitas, J., Leinert, C., \& Köhler, R. 2001, A\&A, 376, 982

Zinnecker, H., \& Mathieu, R. D. (ed.) 2001, IAU Symp. 200, The Formation of Binary Stars 


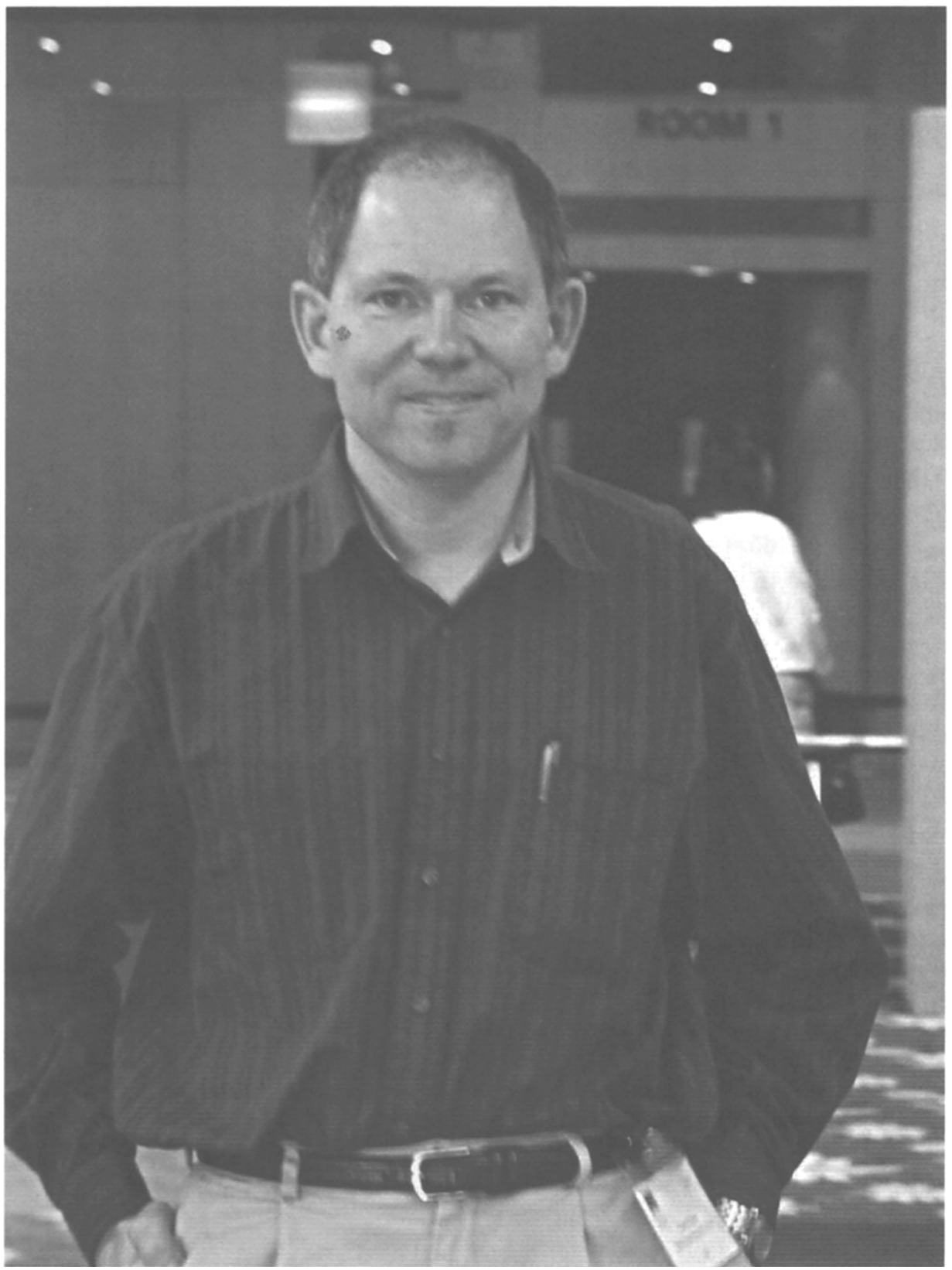

Tom Greene 